\title{
Properties of the Steiner Triple Systems of Order 19
}

\author{
Charles J. Colbourn*
}

School of Computing, Informatics, and Decision Systems Engineering

Arizona State University, Tempe, AZ 85287-8809, U.S.A.

Anthony D. Forbes, Mike J. Grannell, Terry S. Griggs

Department of Mathematics and Statistics, The Open University

Walton Hall, Milton Keynes MK7 6AA, United Kingdom

\section{Petteri Kaski ${ }^{\dagger}$}

Helsinki Institute for Information Technology HIIT

University of Helsinki, Department of Computer Science

P.O. Box 68, 00014 University of Helsinki, Finland

Patric R. J. Östergård

Department of Communications and Networking

Aalto University

P.O. Box 13000, 00076 Aalto, Finland

David A. Pike

Department of Mathematics and Statistics

Memorial University of Newfoundland

St. John's, NL, Canada A1C 5S7

\section{Olli Pottonen}

Department of Communications and Networking

Aalto University

P.O. Box 13000, 00076 Aalto, Finland

Submitted: Sep 22, 2009; Accepted: Jul 1, 2010; Published: Jul 10, 2010

Mathematics Subject Classification: 05B07

${ }^{*}$ Supported in part by DOD Grant N00014-08-1-1070.

†Supported by the Academy of Finland, Grant No. 117499.

${ }^{\ddagger}$ Supported in part by the Academy of Finland, Grants No. 107493, 110196, 130142, 132122.

${ }^{\S}$ Supported in part by CFI, IRIF and NSERC.

`Current address: Finnish Defence Forces Technical Research Centre, P.O. Box 10, 11311 Riihimäki, Finland. Supported by the Graduate School in Electronics, Telecommunication and Automation, by the Nokia Foundation and by the Academy of Finland, Grant No. 110196. 


\begin{abstract}
Properties of the 11084874829 Steiner triple systems of order 19 are examined. In particular, there is exactly one 5-sparse, but no 6-sparse, STS(19); there is exactly one uniform STS(19); there are exactly two STS(19) with no almost parallel classes; all STS(19) have chromatic number 3; all have chromatic index 10, except for 4075 designs with chromatic index 11 and two with chromatic index 12; all are 3-resolvable; and there are exactly two 3-existentially closed STS(19).
\end{abstract}

Keywords: automorphism, chromatic index, chromatic number, configuration, cycle structure, existential closure, independent set, partial parallel class, rank, Steiner triple system of order 19 .

\title{
1 Introduction
}

A Steiner triple system $(\mathrm{STS})$ is a pair $(X, \mathcal{B})$, where $X$ is a finite set of points and $\mathcal{B}$ is a collection of 3 -subsets of points, called blocks or triples, with the property that every 2 -subset of points occurs in exactly one block. The size of the point set, $v:=|X|$, is the order of the design, and an STS of order $v$ is commonly denoted by $\operatorname{STS}(v)$. Steiner triple systems form perhaps the most fundamental family of combinatorial designs; it is well known that they exist exactly for orders $v \equiv 1,3(\bmod 6)$ [31].

Two $\operatorname{STS}(v)$ are isomorphic if there is a bijection between their point sets that maps blocks onto blocks. Denoting the number of isomorphism classes of $\operatorname{STS}(v)$ by $N(v)$, we have $N(3)=1, N(7)=1, N(9)=1, N(13)=2$ and $N(15)=80$. Indeed, due to their relatively small number, the STSs up to order 15 have been studied in detail and are rather well understood. An extensive study of their properties was carried out by Mathon, Phelps and Rosa in the early 1980s [35].

For the next admissible parameter, we have $N(19)=11084874829$, obtained in [26]. Of course, this huge number prohibits a discussion of each individual design. Because the designs are publicly available in compressed form [28], however, examination of some of their properties can be easily automated. Computing resources set a strict limit on what is feasible: one CPU year permits 2.8 milliseconds on average for each design.

Many properties of interest can nonetheless be treated. In Section 2, results, mainly of a computational nature, are presented. They show, amongst other things, that there is exactly one 5-sparse, but no 6-sparse, STS(19); that there is one uniform STS(19); that there are two STS(19) with no almost parallel classes; that all STS(19) have chromatic number 3; that all have chromatic index 10, except for 4075 designs with chromatic index 11 and two with chromatic index 12; that all STS(19) are 3-resolvable; and that there are two 3-existentially closed STS(19). Some tables from the original classification [26] are repeated for completeness. In Section 3, some properties that remain open are mentioned, and the computational resources needed in the current work are briefly discussed. 
Table 1: Automorphism group order

\begin{tabular}{rrrrrrrr}
\hline Aut $\mid$ & $\#$ & $\mid$ Aut $\mid$ & $\#$ & $\mid$ Aut $\mid$ & $\#$ & $\mid$ Aut $\mid$ & $\#$ \\
\hline 1 & 11084710071 & 8 & 101 & 19 & 1 & 96 & 1 \\
2 & 149522 & 9 & 19 & 24 & 11 & 108 & 1 \\
3 & 12728 & 12 & 37 & 32 & 3 & 144 & 1 \\
4 & 2121 & 16 & 13 & 54 & 2 & 171 & 1 \\
6 & 182 & 18 & 11 & 57 & 2 & 432 & 1 \\
\hline
\end{tabular}

\section{Properties}

\subsection{Automorphisms}

The automorphisms and automorphism groups of the STS(19) were studied in [6, 26]; we reproduce the results here (with a correction in our Table 2).

Representing an automorphism as a permutation of the points, the nonidentity automorphisms can be divided into two types based on their order. The automorphisms of prime order have six cycle types

$$
19^{1}, \quad 1^{1} 2^{9}, \quad 1^{1} 3^{6}, \quad 1^{3} 2^{8}, \quad 1^{7} 2^{6}, \quad 1^{7} 3^{4},
$$

and the automorphisms of composite order have nine cycle types

$$
1^{1} 9^{2}, \quad 1^{1} 6^{3}, \quad 1^{1} 3^{2} 6^{2}, \quad 1^{1} 2^{1} 4^{4}, \quad 1^{1} 2^{1} 8^{2}, \quad 1^{3} 8^{2}, \quad 1^{3} 4^{4}, \quad 1^{3} 2^{2} 6^{2}, \quad 1^{3} 2^{2} 4^{3} .
$$

Table 1 gives the order of the automorphism group for each isomorphism class. Tables 2 and 3 partition the possible orders of the automorphism groups into classes based on the types of prime and composite automorphisms that occur in the group. Compared with [26], Table 2 has been corrected by transposing the classes $18 \mathrm{c}$ and $18 \mathrm{~d}$, and the classes $12 \mathrm{a}$ and $12 \mathrm{~b}$ (this correction is incorporated in the table reproduced in [4]).

A list of the 104 STS(19) having an automorphism group of order at least 9 is given in compact notation in the supplement to [6]. Cyclic STS(19) were first enumerated in [1] and 2-rotational ones (automorphism cycle type $1^{1} 9^{2}$ ) in [38]; these systems are listed in [35]. The 184 reverse $\operatorname{STS}(19)$ (automorphism cycle type $1^{1} 2^{9}$ ), together with their automorphism groups, were determined in [10].

In this paper, certain STS(19) are identified as follows: A1-A4 are the cyclic systems as listed in [35]; B1-B10 are the 2-rotational STS(19) as listed in [35]; and S1-S7 are the sporadic STS(19) listed in the Appendix. In addition, an STS(19) can be identified by the order of its automorphism group when this is unique (the listings in [6] are useful for retrieving such designs). Design A4, with an automorphism group of order 171, is both cyclic and 2-rotational and is therefore also listed as B8 in [35]; it is the Netto triple system [39]. A reader interested in copies of STS(19) that are not included among the sporadic examples here will apparently need to carry out some computational work, perhaps utilizing the catalogue from [28] - the authors of the current work are glad to provide consultancy for such an endeavour. 
Table 2: Automorphisms (prime order)

\begin{tabular}{|c|c|c|c|c|c|c|c|c|}
\hline Order & Class & $19^{1}$ & $1^{1} 2^{9}$ & $1^{1} 3^{6}$ & $1^{3} 2^{8}$ & $1^{7} 2^{6}$ & $1^{7} 3^{4}$ & $\#$ \\
\hline 432 & & & & $*$ & $*$ & $*$ & $*$ & 1 \\
\hline 171 & & * & & $*$ & & & & 1 \\
\hline 144 & & & & $*$ & $*$ & $*$ & & 1 \\
\hline 108 & & & & $*$ & $*$ & $*$ & $*$ & 1 \\
\hline 96 & & & & $*$ & $*$ & $*$ & & 1 \\
\hline 57 & & $*$ & & * & & & & 2 \\
\hline 54 & & & & $*$ & & $*$ & $*$ & 2 \\
\hline 32 & & & & & $*$ & $*$ & & 3 \\
\hline 24 & & & & * & $*$ & $*$ & & 11 \\
\hline 19 & & $*$ & & & & & & 1 \\
\hline \multirow[t]{4}{*}{18} & $a$ & & $*$ & $*$ & & & & 1 \\
\hline & b & & & * & $*$ & & * & 2 \\
\hline & c & & & * & & $*$ & * & 6 \\
\hline & d & & & $*$ & & $*$ & & 2 \\
\hline 16 & & & & & $*$ & $*$ & & 13 \\
\hline \multirow[t]{4}{*}{12} & $a$ & & & * & * & $*$ & & 8 \\
\hline & b & & & $*$ & $*$ & & & 7 \\
\hline & $\mathrm{c}$ & & & $*$ & & * & & 12 \\
\hline & d & & & & $*$ & $*$ & * & 10 \\
\hline 9 & & & & $*$ & & & & 19 \\
\hline \multirow[t]{2}{*}{8} & $a$ & & & & $*$ & * & & 84 \\
\hline & b & & & & $*$ & & & 17 \\
\hline \multirow[t]{5}{*}{6} & $\mathrm{a}$ & & * & $*$ & & & & 14 \\
\hline & b & & & $*$ & $*$ & & & 14 \\
\hline & c & & & $*$ & & $*$ & & 116 \\
\hline & d & & & & $*$ & & $*$ & 10 \\
\hline & e & & & & & $*$ & * & 28 \\
\hline \multirow[t]{3}{*}{4} & $\mathrm{a}$ & & & & $*$ & $*$ & & 839 \\
\hline & b & & & & $*$ & & & 662 \\
\hline & c & & & & & $*$ & & 620 \\
\hline \multirow[t]{2}{*}{3} & $\mathrm{a}$ & & & * & & & & 12664 \\
\hline & b & & & & & & $*$ & 64 \\
\hline \multirow[t]{3}{*}{2} & $\mathrm{a}$ & & * & & & & & 169 \\
\hline & b & & & & $*$ & & & 78961 \\
\hline & c & & & & & $*$ & & 70392 \\
\hline \# & & 4 & 184 & 12885 & 80645 & 72150 & 124 & 164758 \\
\hline
\end{tabular}


Table 3: Automorphisms (composite order)

\begin{tabular}{|c|c|c|c|c|c|c|c|c|c|c|}
\hline Class & $1^{1} 9^{2}$ & $1^{1} 6^{3}$ & $1^{1} 3^{2} 6^{2}$ & $1^{1} 2^{1} 4^{4}$ & $1^{1} 2^{1} 8^{2}$ & $1^{3} 8^{2}$ & $1^{3} 4^{4}$ & $1^{3} 2^{2} 6^{2}$ & $1^{3} 2^{2} 4^{3}$ & \# \\
\hline 432 & & & $*$ & & & $*$ & $*$ & $*$ & & 1 \\
\hline 171 & $*$ & & & & & & & & & 1 \\
\hline 144 & & & $*$ & $*$ & $*$ & & $*$ & & & 1 \\
\hline 108 & & & $*$ & & & & & $*$ & & 1 \\
\hline 96 & & & $*$ & & & & * & & & 1 \\
\hline 57 & & & & & & & & & & 2 \\
\hline 54 & & & $*$ & & & & & & & 2 \\
\hline 32 & & & & $*$ & & & $*$ & & & 3 \\
\hline 24 & & & $*$ & & & & & & & 11 \\
\hline 19 & & & & & & & & & & 1 \\
\hline $18 \mathrm{a}$ & & $*$ & & & & & & & & 1 \\
\hline $18 \mathrm{~b}$ & & & & & & & & $*$ & & 2 \\
\hline $18 \mathrm{c}$ & & & * & & & & & & & 6 \\
\hline $18 \mathrm{~d}$ & & & $*$ & & & & & & & 2 \\
\hline 16 & & & & $*$ & $*$ & & $*$ & & & 5 \\
\hline 16 & & & & $*$ & & & & & & 6 \\
\hline 16 & & & & & & * & * & & & 1 \\
\hline 16 & & & & & & & $*$ & & & 1 \\
\hline $12 \mathrm{a}$ & & & * & & & & & & & 8 \\
\hline $12 \mathrm{~b}$ & & & & & & & & & & 7 \\
\hline $12 \mathrm{c}$ & & & & & & & & & & 12 \\
\hline $12 \mathrm{~d}$ & & & & & & & & $*$ & & 10 \\
\hline 9 & $*$ & & & & & & & & & 9 \\
\hline 9 & & & & & & & & & & 10 \\
\hline $8 a$ & & & & & & & * & & & $\begin{array}{r}2 \\
82\end{array}$ \\
\hline \multirow[t]{3}{*}{$8 \mathrm{~b}$} & & & & $*$ & & & $*$ & & & 5 \\
\hline & & & & & * & & $*$ & & & 10 \\
\hline & & & & & & $*$ & $*$ & & & 2 \\
\hline $6 \mathrm{a}$ & & $*$ & & & & & & & & 14 \\
\hline $6 \mathrm{~b}$ & & & & & & & & & & 14 \\
\hline $6 c$ & & & $*$ & & & & & & & 104 \\
\hline & & & & & & & & & & 12 \\
\hline $6 \mathrm{~d}$ & & & & & & & & $*$ & & 10 \\
\hline $6 \mathrm{e}$ & & & & & & & & & & 28 \\
\hline $4 a$ & & & & & & & & & & 839 \\
\hline \multirow[t]{3}{*}{$4 b$} & & & & $*$ & & & & & & 498 \\
\hline & & & & & & & * & & & 153 \\
\hline & & & & & & & & & * & $\begin{array}{l}11 \\
48\end{array}$ \\
\hline $4 \mathrm{c}$ & & & & & & & & & & 572 \\
\hline$\#$ & 10 & 15 & 137 & 518 & 16 & 4 & 185 & 24 & 48 & \\
\hline
\end{tabular}


Table 4: Number of subsystems

\begin{tabular}{rrrrrr}
\hline $\operatorname{STS}(7)$ & STS(9) & $\#$ & STS(7) & STS(9) & $\#$ \\
\hline 0 & 0 & 10997902498 & 3 & 1 & 45 \\
0 & 1 & 270784 & 4 & 0 & 2449 \\
1 & 0 & 86101058 & 4 & 1 & 25 \\
1 & 1 & 12956 & 6 & 0 & 75 \\
2 & 0 & 572471 & 6 & 1 & 5 \\
2 & 1 & 641 & 12 & 0 & 2 \\
3 & 0 & 11819 & 12 & 1 & 1 \\
\hline
\end{tabular}

\subsection{Subsystems and Ranks}

A subsystem in an STS is a subset of blocks that forms an STS on a subset of the points. A subsystem in an $\operatorname{STS}(v)$ has order at most $(v-1) / 2$; hence a subsystem in an $\operatorname{STS}(19)$ has order 3, 7 or 9 . Moreover, the intersection of two subsystems is a subsystem. It follows that each STS(19) has at most one subsystem of order 9, with equality for 284457 isomorphism classes [42]. The number of subsystems of each order in each isomorphism class was determined in [29] and these results are collected in Table 4. The STS(19) with 12 subsystems of order 7 and 1 subsystem of order 9 is the system having an automorphism group of order 432, and the other two STS(19) with 12 subsystems of order 7 are the systems having automorphism groups of orders 108 and 144.

The rank of an STS is the linear rank of its point-block incidence matrix over GF(2). In this setting, a nonempty set of points is (linearly) dependent if every block intersects the set in an even number of points. Counting the point-block incidences in a dependent set in two different ways, one finds that a dependent set necessarily consists of $(v+1) / 2$ points so that its complement is the point set of a subsystem of order $(v-1) / 2$. An in-depth study of the rank of STSs has been carried out in [11].

In particular, for $v=19$ there is at most one dependent set, with equality if and only if there exists a subsystem of order 9. It follows that the rank of an STS(19) is 18 if there exists a subsystem of order 9 (284457 isomorphism classes) and 19 otherwise (11084590372 isomorphism classes).

The rank over $\mathrm{GF}(2)$ gives the dimension of the binary code generated by the (rows or columns of) the incidence matrix. The code generated by the rows of a point-block incidence matrix is the point code of the STS. There exist nonisomorphic STS(19) that have equivalent point codes [27].

\subsection{Small Configurations}

A configuration $\mathcal{C}$ in an $\operatorname{STS}(X, \mathcal{B})$ is a subset of blocks $\mathcal{C} \subseteq \mathcal{B}$. Small configurations in STSs have been studied extensively; see [8, Chapter 13], [17] and [19]. The number of any configuration of size at most 3 is a function of the order of the STS. We address small configurations with some particular properties. 
A configuration $\mathcal{C}$ with $|\mathcal{C}|=\ell$ and $\left|\cup_{C \in \mathcal{C}} C\right|=k$ is a $(k, \ell)$-configuration. A configuration is even if each of its points occurs in an even number of blocks. If no point of a configuration occurs in exactly one block, then the configuration is full.

The only even (and only full) configuration of size 4 is the Pasch configuration, the $(6,4)$-configuration depicted in Figure 1. The numbers of Pasch configurations in the STS(19) were tabulated in [26]; for completeness, we repeat the result in Table 5.

Table 5: Number of Pasches

\begin{tabular}{rrrrrrrr}
\hline Pasch & \# & Pasch & \# & Pasch & \# & Pasch & \# \\
\hline 0 & 2591 & 17 & 954710609 & 34 & 2190166 & 51 & 366 \\
1 & 35758 & 18 & 845596671 & 35 & 1301951 & 52 & 482 \\
2 & 263646 & 19 & 716603299 & 36 & 775233 & 53 & 78 \\
3 & 1315161 & 20 & 583321976 & 37 & 452306 & 54 & 278 \\
4 & 4958687 & 21 & 457755898 & 38 & 267642 & 55 & 69 \\
5 & 15095372 & 22 & 347324307 & 39 & 152122 & 56 & 137 \\
6 & 38481050 & 23 & 255589428 & 40 & 92056 & 57 & 24 \\
7 & 84328984 & 24 & 182938899 & 41 & 51019 & 58 & 104 \\
8 & 162045054 & 25 & 127614183 & 42 & 31587 & 59 & 6 \\
9 & 276886518 & 26 & 87003115 & 43 & 16974 & 60 & 41 \\
10 & 426050673 & 27 & 58052942 & 44 & 11827 & 62 & 47 \\
11 & 596271997 & 28 & 38010203 & 45 & 6008 & 64 & 3 \\
12 & 765958741 & 29 & 24457073 & 46 & 4629 & 66 & 18 \\
13 & 910510124 & 30 & 15492114 & 47 & 2151 & 70 & 5 \\
14 & 1008615673 & 31 & 9663499 & 48 & 2099 & 78 & 2 \\
15 & 1047850033 & 32 & 5956712 & 49 & 724 & 84 & 3 \\
16 & 1027129335 & 33 & 3623356 & 50 & 991 & & \\
\hline
\end{tabular}

Three STS(19) with 84 Pasch configurations were found in [23]. Indeed, 84 is the maximum possible number of Pasch configurations and the list of such STS(19) in [23] is complete. The three systems are those having automorphism groups of order 108, 144 and 432, also encountered in Section 2.2.

Replacing the blocks of a Pasch configuration, say $\mathcal{P}=\{\{a, b, c\},\{a, y, z\},\{x, b, z\}$, $\{x, y, c\}\}$, by the blocks of $\mathcal{P}^{\prime}=\{\{x, y, z\},\{x, b, c\},\{a, y, c\},\{a, b, z\}\}$ transforms an STS into another STS. This operation is a Pasch switch. All but one of the 80 isomorphism classes of STS(15) contain at least one Pasch configuration. Any one of these can be transformed to any other by some sequence of Pasch switches [16, 22]. A natural question is whether the same is true for the STS(19), that is, if each STS(19) containing at least one Pasch configuration can be transformed to any other such design via Pasch switches. The answer is in the negative.

In [21] the concept of twin Steiner triple systems was introduced. These are two STSs each of which contains precisely one Pasch configuration that when switched produces the other system. If in addition the twin systems are isomorphic we have identical twins. In 
[20] nine pairs of twin STS(19) are given. By examining all STS(19) containing a single Pasch configuration, we have established that there are in total 126 pairs of twins, but no identical twins.

We also consider STSs that contain precisely two Pasch configurations, say $\mathcal{P}$ and $\mathcal{Q}$, such that when $\mathcal{P}$ (respectively $\mathcal{Q}$ ) is switched what is obtained is an STS containing just one Pasch configuration $\mathcal{P}^{\prime}$ (respectively $\mathcal{Q}^{\prime}$ ). There are precisely 9 such systems. In every case the two single Pasch systems obtained by the Pasch switches are nonisomorphic. One such system is $\mathrm{S} 1$ (in the Appendix).

For size 6, there are two even configurations, known as the grid and the prism (or double triangle); these $(9,6)$-configurations are depicted in Figure 1.

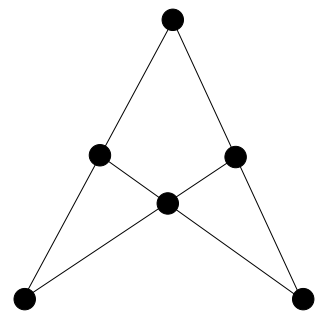

Pasch

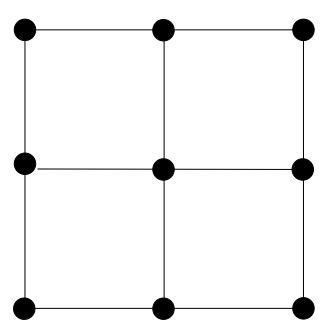

Grid

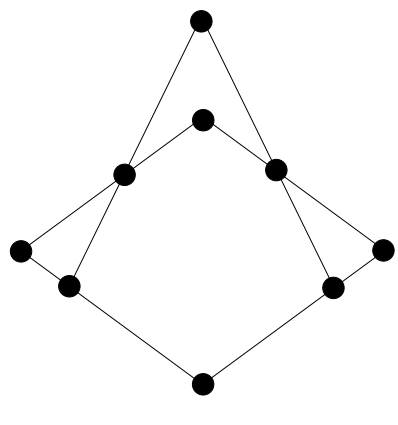

Prism

Figure 1: The even configurations of size at most 6

Every STS contains an even configuration of size at most 8, see [15]. However, no STS(19) missing either a grid or a prism was known. Indeed, a complete enumeration of grids and prisms establishes that there is no such STS(19). The distribution of the numbers of grids is shown in Table 9 and that for prisms in Table 10. The smallest number of grids in an STS(19) is 21 (design S4) and the largest is 384 (the STS(19) with automorphism group order 432). The smallest number of prisms is 171 (design A4) and the largest is 1152 (the designs with automorphism group orders 108, 144 and 432). In particular, then, every STS(19) contains both even $(9,6)$-configurations.

An STS is $k$-sparse if it does not contain any $(n+2, n)$-configuration for any $4 \leqslant$ $n \leqslant k$. In studying $k$-sparse systems it suffices to focus on full configurations, because an $(n+2, n)$-configuration that is not full contains an $(n+1, n-1)$-configuration. Because $k$-sparse STS(19) with $k \geqslant 4$ are anti-Pasch, one could simply check the 2591 anti-Pasch STS(19). A more extensive tabulation of small $(n+2, n)$-configurations was carried out in this work.

There is one full $(7,5)$-configuration (the mitre) and two full $(8,6)$-configurations, known as the hexagon (or 6-cycle) and the crown. These are drawn in Figure 2, and their numbers are presented in Tables 11, 12 and 13.

The existence of a 5-sparse STS(19) was known [7]. By Table 11 there are exactly four nonisomorphic anti-mitre STS(19). Moreover, by Tables 12 and 13 there is a unique STS(19) with no hexagon and exactly four with no crown. Considering the intersections 


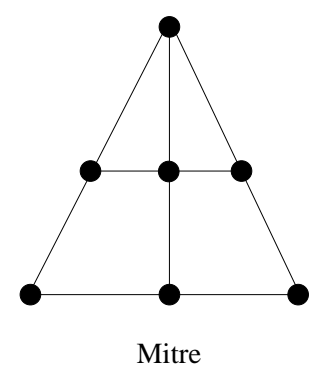

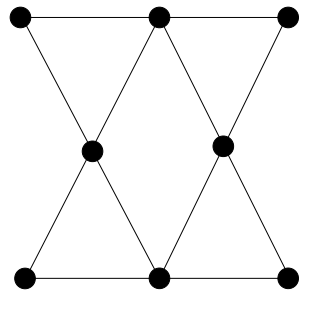

Hexagon

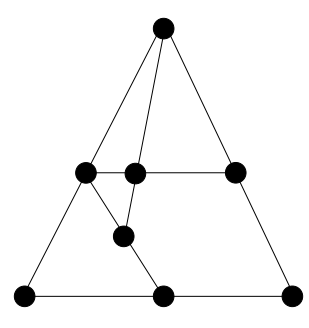

Crown

Figure 2: The full $(7,5)$ - and $(8,6)$-configurations

of the classes of STS(19) with these properties, and the anti-Pasch ones, only two STS(19) are in more than one of the classes: one has no Pasch and no mitre, and one has no Pasch and no crown.

Theorem 1. The numbers of 4-sparse, 5-sparse and 6-sparse STS(19) are 2591, 1 and 0 , respectively.

The unique 5-sparse - that is, anti-Pasch and anti-mitre - STS(19) is A4. The unique STS(19) having no Pasch and no crown is A2, and the unique STS(19) with no hexagon is S5. The other three anti-mitre systems are B4, S6 and A3, and the other three anticrown systems are those with automorphism group orders 108, 144 and 432. The largest number of mitres, hexagons and crowns in an STS(19) is 144 (for the three STS(19) with automorphism group orders 108, 144 and 432), 171 (for A4) and 314 (for S7), respectively.

\subsection{Cycle Structure and Uniform Systems}

Any two distinct points $x, y \in X$ of an STS determine a cycle graph in the following way. The points $x, y$ occur in a unique block $\{x, y, z\}$. The cycle graph has one vertex for each point in $X \backslash\{x, y, z\}$ and an edge between two vertices if and only if the corresponding points occur together with $x$ or $y$ in a block.

A cycle graph of an STS is 2-regular and consists of a set of cycles of even length. Hence they can be specified as integer partitions of $v-3$ using even integers greater than or equal to 4 . For $v=19$, the possible partitions are $l_{1}=4+4+4+4, l_{2}=4+4+8, l_{3}=4+6+6$, $l_{4}=4+12, l_{5}=6+10, l_{6}=8+8$ and $l_{7}=16$. The cycle vector of an STS is a tuple showing the distribution of the cycle graphs; for STS(19) we have $\left(a_{1}, a_{2}, a_{3}, a_{4}, a_{5}, a_{6}, a_{7}\right)$ with $\sum_{i=1}^{7} a_{i}=\left(\begin{array}{c}19 \\ 2\end{array}\right)=171$, where $a_{i}$ denotes the number of occurrences of the partition $l_{i}$.

The cycle vector $(0,0,0,0,0,0,171)$ is of particular interest; an STS all of whose cycle graphs consist of a single cycle is perfect. It is known [25] that there is no perfect STS(19). A more general family consists of the STSs with $a_{i}=\left(\begin{array}{l}v \\ 2\end{array}\right)$ for some $i$; such STSs are uniform. Uniform STS(19) are known to exist [39]. 
An extensive investigation of the cycle vectors of STS(19) was carried out. The results are summarized in Table 6, where the designs are grouped according to the support of the cycle vector, that is, $\left\{i: a_{i} \neq 0\right\}$. Only 28 out of 128 possible combinations of cycle graphs are actually realised.

Table 6: Combinations of cycle graphs

\begin{tabular}{lrlrlr}
\hline Type & $\#$ & Type & $\#$ & Type & $\#$ \\
\hline 5 & 1 & 3567 & 125 & 24567 & 75786636 \\
57 & 5 & 4567 & 5009893 & 34567 & 174351058 \\
134 & 3 & 12347 & 39 & 123457 & 51146 \\
347 & 1 & 12457 & 56 & 123467 & 15 \\
357 & 1 & 12467 & 1 & 124567 & 8658874 \\
457 & 17 & 13457 & 89 & 134567 & 11039468 \\
567 & 2585 & 13467 & 2 & 234567 & 8685731027 \\
1347 & 5 & 14567 & 135588 & 1234567 & 2124060807 \\
2457 & 255 & 23457 & 46863 & & \\
3457 & 259 & 23567 & 10 & \\
\hline
\end{tabular}

The main observation from Table 6 is the following.

Theorem 2. There is exactly one uniform STS(19).

The following conclusions can also be drawn from Table 6. The anti-Pasch systems are one with cycle graph 5 ; five with cycle graphs 5 and 7 ; and 2585 with cycle graphs 5 , 6 and 7. The unique 6-cycle-free system has cycle graphs 1,2, 4, 6 and 7 . The numbers of $k$-cycle-free systems for $k=4,6,8,10,12$ and 16 are 2591, 1, 381, 66, 2727 and 4, respectively. The unique uniform $\operatorname{STS}(19)$ is the 5-sparse system A4 of Theorem 1.

\subsection{Independent Sets}

An independent set $I \subseteq X$ in a Steiner triple system $(X, \mathcal{B})$ is a set of points with the property that no block of $\mathcal{B}$ is contained in $I$. A maximum independent set is an independent set of maximum size. There exists an STS(19) that contains a maximum independent set of size $m$ if and only if $m \in\{7,8,9,10\}$, and $m=10$ arises precisely when the design contains a subsystem of order 9 ; see [8, Chapter 17]. The following theorem collects the results of a complete determination.

Theorem 3. The numbers of STS(19) with maximum independent set size 7, 8, 9 and 10 are 2, 10133102887,951487483 and 284 457, respectively.

The two systems that have maximum independent set of size 7 are the (cyclic) systems A2 and A4. 


\subsection{Chromatic Number}

A colouring of a Steiner triple system $(X, \mathcal{B})$ is a partition of $X$ into independent sets. A partition of $X$ into $k$ independent sets is a $k$-colouring. The chromatic number of an STS is the smallest integer $k$ such that the STS has a $k$-colouring, and corresponding colourings are optimal. Designs with a unique optimal colouring have been termed uniquely colourable [41]. A colouring is equitable if the cardinalities of the colour classes differ by at most one. An STS is $k$-balanced if every $k$-colouring is equitable.

No $\operatorname{STS}(v)$ with $v>3$ is 2-chromatic [40]. Moreover, every STS(19) is 4-colourable [13, Theorem 6.1]; see also [24, Theorem 5]. Consequently, the chromatic number of any $\mathrm{STS}(19)$ is either 3 or 4 . No STS(19) with chromatic number 4 was known; indeed as we see next, none exists. An exhaustive search establishes the following.

Theorem 4. Every STS(19) is 3-chromatic. More specifically,

(i) every $\operatorname{STS}(19)$ has a 3 -colouring with colour class sizes $(7,7,5)$ and

(ii) every $\mathrm{STS}(19)$ except for designs $\mathrm{A} 2$ and A4 has a 3-colouring with colour class sizes $(8,6,5)$.

Next we show that Theorem 4 completes the determination of the combinations of 3-colouring patterns that can occur in an $\operatorname{STS}(19)$. For a given 3-colouring of an $\operatorname{STS}(19)$, let the colour classes be $\left(C_{1}, C_{2}, C_{3}\right)$. Let $c_{i}=\left|C_{i}\right|$ for $1 \leqslant i \leqslant 3$. Without loss of generality suppose that $c_{1} \geqslant c_{2} \geqslant c_{3}$, and denote the pattern of colour class sizes by the corresponding integer triple $\left(c_{1}, c_{2}, c_{3}\right)$. Informally, we refer to the colour classes $C_{1}, C_{2}, C_{3}$ as red, yellow and blue. It is shown in [12, Section 2.4] and [13] that any 3-colouring of an STS(19) must have one of the six patterns

$$
(7,6,6),(7,7,5),(8,6,5),(8,7,4),(9,5,5),(9,6,4),
$$

and that certain reductions are possible.

Lemma 1. An STS(19) that has a 3-colouring with colour class sizes

(i) $(7,7,5)$ also has one with sizes $(7,6,6)$,

(ii) $(8,6,5)$ either has one with sizes $(7,7,5)$ or one with sizes $(7,6,6)$,

(iii) $(8,7,4)$ also has one with sizes $(7,7,5)$,

(iv) $(9,5,5)$ either has one with sizes $(9,6,4)$ or one with sizes $(8,6,5)$,

(v) $(9,6,4)$ also has one with sizes $(8,6,5)$,

(vi) $(8,7,4)$ also has one with sizes $(8,6,5)$,

(vii) $(9,5,5)$ also has one with sizes $(8,6,5)$,

(viii) $(9,6,4)$ also has one with sizes $(9,5,5)$,

(ix) $(9,6,4)$ also has one with sizes $(8,7,4)$. 
Proof. For $(i)-(v)$, see [12, Section 2.4] or [13, Section 4]. It remains only to prove (vi)-(ix).

Let $x_{i j k}, 1 \leqslant i \leqslant j \leqslant k$, denote the number of blocks containing points belonging to colour classes $C_{i}, C_{j}$ and $C_{k}$, with appropriate multiplicities. Thus, for example, $x_{122}$ is the number of blocks that contain a red point and two yellow points. Write $x$ for $x_{223}$. As in the proof of $[12$, Theorem 2.4.1] we can construct the following table by a straightforward computation.

\begin{tabular}{cccccccc}
\hline$\left(c_{1}, c_{2}, c_{3}\right)$ & $x_{122}$ & $x_{133}$ & $x_{112}$ & $x_{113}$ & $x_{223}$ & $x_{233}$ & $x_{123}$ \\
\hline$(7,6,6)$ & $15-x$ & $x$ & $3+x$ & $18-x$ & $x$ & $15-x$ & 6 \\
$(7,7,5)$ & $21-x$ & $x-5$ & $1+x$ & $20-x$ & $x$ & $15-x$ & 5 \\
$(8,6,5)$ & $15-x$ & $x-3$ & $7+x$ & $21-x$ & $x$ & $13-x$ & 4 \\
$(8,7,4)$ & $21-x$ & $x-7$ & $6+x$ & $22-x$ & $x$ & $13-x$ & 2 \\
$(9,5,5)$ & $10-x$ & $x-2$ & $12+x$ & $24-x$ & $x$ & $12-x$ & 1 \\
$(9,6,4)$ & $15-x$ & $x-6$ & $12+x$ & $24-x$ & $x$ & $12-x$ & 0 \\
\hline
\end{tabular}

Suppose we have an $(8,7,4) 3$-colouring of an $\operatorname{STS}(19)$. Then $x \geqslant 7$ since $x_{133}=$ $x-7 \geqslant 0$. Moreover, $x_{233}=13-x \leqslant 6$. Therefore we can find a yellow point to change to blue without creating a blue-blue-blue block. This proves (vi).

Suppose we have a $(9,5,5) 3$-colouring. Since $x_{122}+x_{133}=8<9$ we can find a red point to be changed to either yellow or blue. This proves (vii).

Suppose we have a $(9,6,4) 3$-colouring. If $x_{233}<6$, we can change a yellow point to blue. So we may assume that $x_{233}=6$. Then $x_{133}=x_{123}=0$. Hence each blue point occurs exactly three times in the yellow-blue-blue blocks and paired with three yellow points. So each blue point must occur paired with three yellow points in yellow-yellowblue blocks. This is impossible; hence (viii) is proved.

Again, suppose we have a $(9,6,4) 3$-colouring. If $x_{122}<9$, we can change a red point to yellow. Otherwise $x_{122} \geqslant 9$. This forces $x=x_{223}=x_{233}=6$ and $x_{133}=x_{123}=0$, which is impossible by the same argument as in the proof of (viii). This proves (ix).

The main result of this section is a straightforward consequence of Theorem 4 and Lemma 1.

Theorem 5. Any STS(19) is 3-colourable with one of the following six combinations of 3-colouring patterns:

$$
\begin{aligned}
& \mathcal{C}_{1}=\{(7,6,6),(7,7,5)\} \\
& \mathcal{C}_{2}=\{(7,6,6),(7,7,5),(8,6,5)\}, \\
& \mathcal{C}_{3}=\{(7,6,6),(7,7,5),(8,6,5),(8,7,4)\}, \\
& \mathcal{C}_{4}=\{(7,6,6),(7,7,5),(8,6,5),(9,5,5)\}, \\
& \mathcal{C}_{5}=\{(7,6,6),(7,7,5),(8,6,5),(8,7,4),(9,5,5)\}, \\
& \mathcal{C}_{6}=\{(7,6,6),(7,7,5),(8,6,5),(8,7,4),(9,5,5),(9,6,4)\} .
\end{aligned}
$$


The first combination in Theorem $5,\{(7,6,6),(7,7,5)\}$, occurs in only two STS(19), both of which are cyclic; in fact these are the two exceptions of Theorem 4(ii), systems A2 and A4. The other two cyclic STS(19), A1 and A3, have the colouring pattern combination $\{(7,6,6),(7,7,5),(8,6,5)\}$. It is easy to find examples exhibiting each of the remaining combinations.

We are now able to answer the open problem of whether there exists a 3-balanced STS(19) [13, Problem 1]. By [13, Theorem 4.1] and Theorems 4 and 5 we immediately get the following.

Corollary 1. Every STS(19) is 3-chromatic and has an equitable 3-colouring. There exists no 3-balanced STS(19).

In a separate computation we obtained the frequency of occurrence of each combination of 3-colouring patterns. We also obtained information concerning the size of maximum independent sets. Our results are presented in Table 7 in the form of a two-way frequency table of maximum independent set size against combinations of 3-colouring patterns $\mathcal{C}_{i}$ as defined in Theorem 5 . The cell in row $\mathcal{C}_{i}$, column $j$ gives the number of STS(19) that have 3-colouring pattern combination $\mathcal{C}_{i}$ and maximum independent set size $j$. Observe that the total count for size 10 is in agreement with [42], and it is worth pointing out that the zero entries in rows $\mathcal{C}_{2}$ to $\mathcal{C}_{6}$ can be deduced by elementary arguments without the need for any extensive computation. In particular, it is not difficult to show that an independent set of size 10 excludes the possibility of a $(9,5,5) 3$-colouring.

Table 7: Colourings and maximum independent sets

\begin{tabular}{crrrrr}
\hline Colouring & 7 & 8 & 9 & 10 & Total \\
\hline $\mathcal{C}_{1}$ & 2 & 0 & 0 & 0 & 2 \\
$\mathcal{C}_{2}$ & 0 & 53680512 & 2650830 & 1241 & 56332583 \\
$\mathcal{C}_{3}$ & 0 & 10079422375 & 421936849 & 283216 & 10501642440 \\
$\mathcal{C}_{4}$ & 0 & 0 & 2912144 & 0 & 2912144 \\
$\mathcal{C}_{5}$ & 0 & 0 & 464995662 & 0 & 464995662 \\
$\mathcal{C}_{6}$ & 0 & 0 & 58991998 & 0 & 58991998 \\
\hline Total & 2 & 10133102887 & 951487483 & 284457 & 11084874829 \\
\hline
\end{tabular}

\subsection{Almost Parallel Classes}

A set of nonintersecting blocks that do not contain all points of the design is a partial parallel class, and a partial parallel class with $\lfloor v / 3\rfloor$ blocks is an almost parallel class. Consequently, six nonintersecting blocks of an STS(19) form an almost parallel class. For each STS(19) we determined the total number of almost parallel classes in the following way.

For each STS(19), the point to be missed by the almost parallel class is specified, after which the problem of finding the almost parallel classes can be formulated as instances 
of the exact cover problem. In the exact cover problem, a set $U$ and a collection $\mathcal{S}$ of subsets of $U$ are given, and one wants to determine (one or all) partitions of $U$ using sets from $\mathcal{S}$. To solve instances of the exact cover problem, the libexact software [30], which implements ideas from work by Knuth [32], was utilized. The results are presented in Table 8.

There is a conjecture that for all $v \equiv 1,3(\bmod 6), v \geqslant 15$, there exists an $\operatorname{STS}(v)$ whose largest partial parallel class has fewer than $\lfloor v / 3\rfloor$ blocks [4, Conjecture 2.86], [8, Conjectures 19.4 and 19.5], [41, Section 3.1]. The results in the current work are in accordance with this conjecture.

In fact, Lo Faro already showed that every STS(19) has a partial parallel class with five blocks [33] and, constructively, that there indeed exists an STS(19) with no almost parallel class [34]. The current work shows that there are exactly two STS(19) with no almost parallel classes. These are A4 and the unique design with automorphism group of order 432. The largest number of almost parallel classes, 182, arises in S3.

A set of blocks of a design with the property that each point occurs in exactly $\alpha$ of these blocks is an $\alpha$-parallel class. A partition of all blocks into $\alpha$-parallel classes is an $\alpha$-resolution, and a design that admits an $\alpha$-resolution is $\alpha$-resolvable. A Steiner triple system whose order $v$ is not divisible by 3 cannot have a (1-)parallel class, but may have a 3-parallel class. The existence of Steiner triple systems of order at least 7 without a 3 -parallel class is an open problem [8, p. 419].

A complete search demonstrates that every STS(19) not only has a 3-parallel class, but a 3-resolution. It is, however, not always the case that every 3-parallel class can be extended to a 3-resolution. That is, some $\operatorname{STS}(19)$ contain a 6-parallel class that is nonseparable, in that it does not further partition into two 3-parallel classes. Using [3], the largest $\alpha$ for which an $\operatorname{STS}(v)$ contains a nonseparable $\alpha$-parallel class is $3,1,3,5$ and 6 for $v=7,9,13,15$ and 19 , respectively.

\subsection{Chromatic Index}

While the chromatic number concerns colouring points, the chromatic index concerns colouring blocks. More precisely, the chromatic index of an STS is the smallest number of colours that can be used to colour the blocks so that no two intersecting blocks receive the same colour.

$\operatorname{An} \operatorname{STS}(v)$ is resolvable if and only if its chromatic index is $(v-1) / 2$. Since 19 is not divisible by 3 , there is no resolvable STS(19), and the smallest possible chromatic index for such a design is $\lceil 57 / 6\rceil=10$.

By elementary counting, an STS(19) with chromatic index 10 must have at least 7 disjoint almost parallel classes. Moreover, the chromatic index of an STS(19) with no almost parallel classes is at least $\lceil 57 / 5\rceil=12$. We now describe the computational approach used to show that 10,11 and 12 are the only possible chromatic indices for an $\operatorname{STS}(19)$.

Exact algorithms and greedy algorithms for finding the chromatic index and upper bounds on the chromatic index of STSs were presented in the early 1980s [2, 5]. Now 
Table 8: Number of almost parallel classes

\begin{tabular}{rrrrrrrr}
\hline APC & $\#$ & APC & $\#$ & APC & $\#$ & APC & $\#$ \\
\hline 0 & 2 & 79 & 764738 & 110 & 526902725 & 141 & 43290 \\
36 & 1 & 80 & 1224282 & 111 & 495595995 & 142 & 25609 \\
40 & 1 & 81 & 1924007 & 112 & 458547878 & 143 & 14838 \\
48 & 5 & 82 & 2974055 & 113 & 417254801 & 144 & 8604 \\
50 & 1 & 83 & 4513033 & 114 & 373408256 & 145 & 4827 \\
51 & 1 & 84 & 6737331 & 115 & 328678489 & 146 & 2907 \\
52 & 2 & 85 & 9882490 & 116 & 284606260 & 147 & 1581 \\
54 & 5 & 86 & 14239039 & 117 & 242381171 & 148 & 1028 \\
56 & 14 & 87 & 20170633 & 118 & 203039046 & 149 & 522 \\
57 & 6 & 88 & 28071379 & 119 & 167316900 & 150 & 386 \\
58 & 16 & 89 & 38411235 & 120 & 135654277 & 151 & 210 \\
59 & 6 & 90 & 51637134 & 121 & 108190905 & 152 & 173 \\
60 & 31 & 91 & 68231490 & 122 & 84895844 & 153 & 75 \\
61 & 27 & 92 & 88611342 & 123 & 65517542 & 154 & 85 \\
62 & 58 & 93 & 113110188 & 124 & 49778191 & 155 & 32 \\
63 & 65 & 94 & 141933285 & 125 & 37203375 & 156 & 53 \\
64 & 158 & 95 & 175017943 & 126 & 27381347 & 157 & 6 \\
65 & 225 & 96 & 212214494 & 127 & 19807367 & 158 & 22 \\
66 & 476 & 97 & 252843760 & 128 & 14108068 & 159 & 6 \\
67 & 774 & 98 & 296203531 & 129 & 9891578 & 160 & 24 \\
68 & 1606 & 99 & 341097019 & 130 & 6829506 & 162 & 5 \\
69 & 2801 & 100 & 386153551 & 131 & 4633657 & 164 & 12 \\
70 & 5363 & 101 & 429813668 & 132 & 3105171 & 166 & 3 \\
71 & 9930 & 102 & 470269272 & 133 & 2044697 & 167 & 1 \\
72 & 18098 & 103 & 505968628 & 134 & 1327796 & 168 & 1 \\
73 & 32270 & 104 & 535235668 & 135 & 847519 & 172 & 4 \\
74 & 56959 & 105 & 556712827 & 136 & 536040 & 174 & 4 \\
75 & 98415 & 106 & 569489811 & 137 & 332998 & 180 & 1 \\
76 & 168833 & 107 & 572707805 & 138 & 203608 & 182 & 1 \\
77 & 284405 & 108 & 566389062 & 139 & 123411 & & \\
78 & 470557 & 109 & 550847618 & 140 & 74672 & & \\
\hline & & & & & & &
\end{tabular}


modern algorithms for finding colourings and chromatic numbers of graphs can be used to determine the chromatic number of the line graph of the design, which equals the chromatic index of the design.

To find a 10-colouring, the algorithm starts by finding sets of 7 disjoint almost parallel classes. To do this, for each STS(19), all almost parallel classes are first found (as in Section 2.7). Using these, sets of 7 disjoint ones are obtained by an algorithm for finding cliques in graphs (form one vertex for each almost parallel class and place edges between disjoint classes). The Cliquer software [37] can be utilized to find the cliques. The final step is an exhaustive search for three partial parallel classes to partition the remaining $57-7 \cdot 6=15$ blocks.

A more general exhaustive search algorithm was applied to instances with chromatic index greater than 10. The final result is as follows.

Theorem 6. The numbers of STS(19) that have chromatic index 10, 11 and 12 are 11084870752,4075 and 2, respectively.

Consequently, exactly the two STS(19) with no almost parallel classes (see Section 2.7) have chromatic index 12. Our results are consistent with the observation that no $\operatorname{STS}(v)$ with $v>7$ and chromatic index exceeding the minimum chromatic index by more than 2 is known to exist [8, pp. 366-367], [41, p. 411].

\section{$2.9 \quad$ Existential Closure}

The block intersection graph of an STS has one vertex for each block and an edge between two vertices exactly when the corresponding blocks intersect. A graph $G=(V, E)$ is $n$-existentially closed if for every $n$-element subset $S \subseteq V$ of vertices and for every subset $T \subseteq S$, there exists a vertex $x \notin S$ that is adjacent to every vertex in $T$ and nonadjacent to every vertex in $S \backslash T$.

In [14] $n$-existentially closed block intersection graphs of STSs are studied. The block intersection graph of an $\operatorname{STS}(v)$ is 2-existentially closed if and only if $v \geqslant 13$, it cannot be 4-existentially closed [36, Theorem 1] for any $v$, and the only possible orders for which it can be 3-existentially closed are 19 and 21. In fact, two STS(19) possess 3-existentially closed block intersection graphs [14].

The following result from [14, Theorem 4.1] helps in designing an algorithm for determining whether the block intersection graph of an STS is 3-existentially closed.

Theorem 7. The block intersection graph of an $\operatorname{STS}(v)$ is 3-existentially closed if and only if

(i) the $\operatorname{STS}(v)$ contains no subsystem $\operatorname{STS}(7)$,

(ii) the $\operatorname{STS}(v)$ contains no subsystem $\operatorname{STS}(9)$,

(iii) for every set of three nonintersecting blocks, if $v<19$ there exists a block that intersects none of the three, and if $v \geqslant 19$ there exists a block that intersects all three. 
No STS(19) other than those discovered in [14] is 3-existentially closed.

Theorem 8. The number of 3-existentially closed STS(19) is 2.

The two 3-existentially closed STS(19) are A3 and S2.

\section{Conclusions}

The main aim of the current work has been to compute all kinds of properties of STS(19) and collect them in a single place. However, it is impossible to accomplish this task in an exhaustive manner, so we omit discussion of properties that (1) we do not consider to have large general interest, (2) we are not able to present in a compact manner, or (3) we simply are not able to compute at the present time.

For example, we consider various kinds of colouring problems, such as those studied in $[9,18]$, to be of the first type. Any properties that have been used as invariants for STSs cannot, by definition, be tabulated in a compact way and are of the second type; examples of this type include various forms of so-called trains.

The third type of problems contain some very interesting open problems, including those of determining intersection numbers of STSs, maximal sets of disjoint STSs, and whether all STSs are derived. Further information on these problems can be found in $[4,8]$. For example, just determining whether a single STS is derived remains a major challenge.

The problems were addressed using three different computational environments (in Canada, Finland and Great Britain), so we do not try to give exact details about the computations. The computational resources needed partition the problems roughly into three groups: those taking days or at most a couple of weeks ("easy"), those taking up to a couple of years ("intermediate") and those taking up to ten years ("hard"). These CPU times are roughly the times needed for one core of a "contemporary microprocessor".

The intermediate calculations were those of determining subconfigurations $(10 \mathrm{CPU}$ weeks), determining the almost parallel classes (1.5 CPU years), constructing the frequency table of maximum independent set size against 3-colouring pattern combination (12 CPU weeks), showing existence of 3-parallel classes (7 CPU months) and searching for 3-existentially closed designs (9 CPU months). The only one belonging to the category of hard calculations was the determination of the chromatic indices, which consumed just under 8 CPU years. All remaining calculations were "easy".

\section{Appendix}

We use the same method for compressing STSs as in the supplement to [6]. That is, for the points we use the symbols a-s and represent an STS by a string of 57 symbols $x_{1} x_{2} \cdots x_{57}$. The symbol $x_{i}$ is the largest element in the $i$ th block. The other two symbols in the $i$ th block are the smallest pair of symbols not occurring in earlier blocks under the colexicographic ordering of pairs: a pair $y, z$ with $y<z$ is smaller than a pair $y^{\prime}, z^{\prime}$ with 
$y^{\prime}<z^{\prime}$ iff $z<z^{\prime}$, or $z=z^{\prime}$ and $y<y^{\prime}$. The order of the automorphism group is given after each design.

S1: edgfhghijkllmnljompqporqsnsloqprmrsnnopsrqqprosqsrpsqress (1)

S2: cefggfhijijklmnokppqmrsolrsqnqpsnrmornsoqpsqporpqrsrsqsrs (8)

S3: cefghngjljrikoqplrnqmskmsnonsmrlpmoprqpqosopqsrrpsqqsisrs (3)

S4: cefghigpojlijqmplrqokomsnnqpslrommnsrqprnsoprqsrspqqsrsrs (1)

S5: cefghfgjoiksmrlpnksqkmpsnlrnoqmmnqposrprqoorpqsrspqqrssrs (6)

S6: cefghigomjsinksllsjqkmropnlqrpomnrpqpqornsopqrsrpqsqsrsrs (9)

S7: cefihkgsojosmiqmnrlpjqklospnqlpormprnsprqonsoprqsrqqrssrs (1)

\section{References}

[1] S. Bays, Sur les systèmes cycliques de triples de Steiner, Ann. Sci. Ecole Norm Sup. (3) 40 (1923), 55-96.

[2] C. J. Colbourn, Computing the chromatic index of Steiner triple systems, Comput. J. 25 (1982), 338-339.

[3] C. J. Colbourn, Separations of Steiner triple systems: some questions, Bull. Inst. Combin. Appl. 6 (1992), 53-56.

[4] C. J. Colbourn, Triple systems, in Handbook of Combinatorial Designs, C. J. Colbourn and J. H. Dinitz (Editors), 2nd ed., Chapman \& Hall/CRC, Boca Raton, 2007, pp. $58-71$.

[5] C. J. Colbourn and M. J. Colbourn, Greedy colourings of Steiner triple systems, Ann. Discrete Math. 18 (1983), 201-207.

[6] C. J. Colbourn, S. S. Magliveras and D. R. Stinson, Steiner triple systems of order 19 with nontrivial automorphism group, Math. Comp. 59 (1992), 283-295 and S25-S27.

[7] C. J. Colbourn, E. Mendelsohn, A. Rosa and J. Širáň, Anti-mitre Steiner triple systems, Graphs Combin. 10 (1994), 215-224.

[8] C. J. Colbourn and A. Rosa, Triple Systems, Oxford University Press, Oxford, 1999.

[9] P. Danziger, M. J. Grannell, T. S. Griggs and A. Rosa, On the 2-parallel chromatic index of Steiner triple systems, Australas. J. Combin. 17 (1998), 109-131.

[10] R. H. F. Denniston, Non-isomorphic reverse Steiner triple systems of order 19, Ann. Discrete Math. 7 (1980), 255-264.

[11] J. Doyen, X. Hubaut and M. Vandensavel, Ranks of incidence matrices of Steiner triple systems, Math. Zeitschr. 163 (1978), 251-259.

[12] A. D. Forbes, Configurations and Colouring Problems in Block Designs, Ph.D. Thesis, The Open University, November 2006.

[13] A. D. Forbes, M. J. Grannell and T. S. Griggs, On colourings of Steiner triple systems, Discrete Math. 261 (2003), 255-276. 
[14] A. D. Forbes, M. J. Grannell and T. S. Griggs, Steiner triple systems and existentially closed graphs, Electron. J. Combin. 12 (2005), \#R42 and Corrigendum.

[15] Y. Fujiwara and C. J. Colbourn, A combinatorial approach to X-tolerant compaction circuits, IEEE Trans. Inform. Theory 56 (2010), 3196-3206.

[16] P. B. Gibbons, Computing Techniques for the Construction and Analysis of Block Designs, Ph.D. Thesis, University of Toronto, 1976, Department of Computer Science, University of Toronto, Technical Report \#92, May 1976.

[17] M. J. Grannell and T. S. Griggs, Configurations in Steiner triple systems, in Combinatorial Designs and Their Applications (Milton Keynes, 1997), F. C. Holroyd, K. A. S. Quinn, C. Rowley and B. S. Webb (Editors), Chapman \& Hall/CRC Press, Boca Raton, 1999, pp. 103-126.

[18] M. J. Grannell, T. S. Griggs and R. Hill, The triangle chromatic index of Steiner triple systems, Australas. J. Combin. 23 (2001), 217-230.

[19] M. J. Grannell, T. S. Griggs and E. Mendelsohn, A small basis for four-line configurations in Steiner triple systems, J. Combin. Des. 3 (1994), 51-59.

[20] M. J. Grannell, T. S. Griggs and J. P. Murphy, Equivalence classes of Steiner triple systems, Congr. Numer. 86 (1992), 19-25.

[21] M. J. Grannell, T. S. Griggs and J. P. Murphy, Twin Steiner triple systems, Discrete Math. 167/168 (1997), 341-352.

[22] M. J. Grannell, T. S. Griggs and J. P. Murphy, Switching cycles in Steiner triple systems, Utilitas Math. 56 (1999), 3-21.

[23] B. D. Gray and C. Ramsay, On the number of Pasch configurations in a Steiner triple system, Bull. Inst. Combin. Appl. 24 (1998), 105-112.

[24] P. Horak, On the chromatic number of Steiner triple systems of order 25, Discrete Math. 299 (2005), 120-128.

[25] P. Kaski, Nonexistence of perfect Steiner triple systems of orders 19 and 21, Bayreuth. Math. Schr. 74 (2005), 130-135.

[26] P. Kaski and P. R. J. Östergård, The Steiner triple systems of order 19, Math. Comp. 73 (2004), 2075-2092.

[27] P. Kaski and P. R. J. Östergård, There exist non-isomorphic STS(19) with equivalent point codes, J. Combin. Des. 12 (2004), 443-448.

[28] P. Kaski, P. R. J. Östergård, O. Pottonen and L. Kiviluoto, A catalogue of the Steiner triple systems of order 19, Bull. Inst. Combin. Appl. 57 (2009), 35-41.

[29] P. Kaski, P. R. J. Östergård, S. Topalova and R. Zlatarksi, Steiner triple systems of order 19 and 21 with subsystems of order 7, Discrete Math. 308 (2008), 2732-2741.

[30] P. Kaski and O. Pottonen, libexact User's Guide, Version 1.0, Helsinki Institute for Information Technology HIIT, HIIT Technical Reports 2008-1, 2008.

[31] T. P. Kirkman, On a problem in combinations, Cambridge and Dublin Math. J. 2 (1847), 191-204. 
[32] D. E. Knuth, Dancing links, in: J. Davies, B. Roscoe and J. Woodcock (Eds.), Millennial Perspectives in Computer Science, Palgrave Macmillan, Basingstoke, 2000, pp. 187-214.

[33] G. Lo Faro, On the size of partial parallel classes in Steiner systems STS(19) and STS(27), Discrete Math. 45 (1983), 307-312.

[34] G. Lo Faro, Partial parallel classes in Steiner system S(2,3,19), J. Inform. Optim. Sci. 6 (1985), 133-136.

[35] R. A. Mathon, K. T. Phelps and A. Rosa, Small Steiner triple systems and their properties, Ars Combin. 15 (1983), 3-110; and 16 (1983), 286.

[36] N. A. McKay and D. A. Pike, Existentially closed BIBD block-intersection graphs, Electron. J. Combin. 14 (2007), \#R70.

[37] S. Niskanen and P. R. J. Östergård, Cliquer User's Guide, Version 1.0, Communications Laboratory, Helsinki University of Technology, Technical Report T48, 2003.

[38] K. T. Phelps and A. Rosa, Steiner triple systems with rotational automorphisms, Discrete Math. 33 (1981), 57-66.

[39] R. M. Robinson, The structure of certain triple systems, Math. Comp. 29 (1975), 223-241.

[40] A. Rosa, On the chromatic number of Steiner triple systems, in 1970 Combinatorial Structures and their Applications (Proc. Calgary Internat. Conf., Calgary, Alta., 1969), Gordon and Breach, New York, 1970, pp. 369-371.

[41] A. Rosa and C. J. Colbourn, Colorings of block designs, in Contemporary Design Theory: A Collection of Surveys, J. H. Dinitz and D. R. Stinson (Editors), Wiley, New York, 1992, pp. 401-430.

[42] D. R. Stinson and E. Seah, 284457 Steiner triple systems of order 19 contain a subsystem of order 9, Math. Comp. 46 (1985), 717-729. 
Table 9: Number of grids

\begin{tabular}{rrrrrrrr}
\hline Grid & $\#$ & Grid & $\#$ & Grid & $\#$ & Grid & $\#$ \\
\hline 21 & 1 & 58 & 421406261 & 95 & 5466378 & 132 & 19595 \\
22 & 1 & 59 & 455538873 & 96 & 4452414 & 133 & 17568 \\
23 & 1 & 60 & 483962320 & 97 & 3625512 & 134 & 17390 \\
24 & 6 & 61 & 505587977 & 98 & 2964501 & 135 & 15125 \\
25 & 27 & 62 & 519737441 & 99 & 2419681 & 136 & 14765 \\
26 & 44 & 63 & 525975481 & 100 & 1984363 & 137 & 12845 \\
27 & 156 & 64 & 524399635 & 101 & 1625523 & 138 & 12707 \\
28 & 403 & 65 & 515397821 & 102 & 1340634 & 139 & 10911 \\
29 & 1012 & 66 & 499528245 & 103 & 1103378 & 140 & 10689 \\
30 & 2577 & 67 & 477877986 & 104 & 915322 & 141 & 9228 \\
31 & 6067 & 68 & 451447963 & 105 & 756727 & 142 & 9097 \\
32 & 13721 & 69 & 421183378 & 106 & 629794 & 143 & 7629 \\
33 & 29607 & 70 & 388549216 & 107 & 522121 & 144 & 7495 \\
34 & 62549 & 71 & 354553810 & 108 & 439478 & 145 & 6593 \\
35 & 125648 & 72 & 320163173 & 109 & 365162 & 146 & 6407 \\
36 & 246636 & 73 & 286220933 & 110 & 310349 & 147 & 5325 \\
37 & 461547 & 74 & 253571165 & 111 & 256766 & 148 & 5266 \\
38 & 840481 & 75 & 222621207 & 112 & 219625 & 149 & 4318 \\
39 & 1484562 & 76 & 193840439 & 113 & 183979 & 150 & 4386 \\
40 & 2534581 & 77 & 167454239 & 114 & 157625 & 151 & 3507 \\
41 & 4196398 & 78 & 143611784 & 115 & 133530 & 152 & 3515 \\
42 & 6739474 & 79 & 122366578 & 116 & 115251 & 153 & 2820 \\
43 & 10522877 & 80 & 103592757 & 117 & 97139 & 154 & 2838 \\
44 & 15960510 & 81 & 87177751 & 118 & 85923 & 155 & 2265 \\
45 & 23562586 & 82 & 72978536 & 119 & 72545 & 156 & 2455 \\
46 & 33871296 & 83 & 60813771 & 120 & 65014 & 157 & 1830 \\
47 & 47412716 & 84 & 50428258 & 121 & 55582 & 158 & 1905 \\
48 & 64736436 & 85 & 41665785 & 122 & 50393 & 159 & 1433 \\
49 & 86205567 & 86 & 34306651 & 123 & 43478 & 160 & 1552 \\
50 & 112103389 & 87 & 28141430 & 124 & 40275 & 161 & 1124 \\
51 & 142489811 & 88 & 23037710 & 125 & 34759 & 162 & 1284 \\
52 & 177059163 & 89 & 18809436 & 126 & 32578 & 163 & 913 \\
53 & 215192146 & 90 & 15344880 & 127 & 28746 & 164 & 1010 \\
54 & 256144342 & 91 & 12489931 & 128 & 27080 & 165 & 766 \\
55 & 298709622 & 92 & 10159180 & 129 & 23884 & 166 & 843 \\
56 & 341446147 & 93 & 8261382 & 130 & 23163 & 167 & 557 \\
57 & 382864465 & 94 & 6721096 & 131 & 20281 & 168 & 664 \\
\hline & & & & & & & \\
& & & & &
\end{tabular}


Table 9: Number of grids (cont.)

\begin{tabular}{rrrrrrrr}
\hline Grid & $\#$ & Grid & $\#$ & Grid & $\#$ & Grid & $\#$ \\
\hline 169 & 490 & 194 & 80 & 219 & 2 & 249 & 3 \\
170 & 527 & 195 & 19 & 220 & 23 & 250 & 2 \\
171 & 324 & 196 & 90 & 221 & 2 & 252 & 10 \\
172 & 429 & 197 & 21 & 222 & 14 & 254 & 1 \\
173 & 267 & 198 & 70 & 223 & 5 & 255 & 2 \\
174 & 383 & 199 & 8 & 224 & 33 & 256 & 7 \\
175 & 206 & 200 & 97 & 225 & 5 & 258 & 1 \\
176 & 328 & 201 & 16 & 226 & 8 & 260 & 7 \\
177 & 153 & 202 & 39 & 227 & 5 & 262 & 1 \\
178 & 232 & 203 & 6 & 228 & 31 & 264 & 8 \\
179 & 126 & 204 & 79 & 229 & 2 & 267 & 2 \\
180 & 223 & 205 & 5 & 230 & 4 & 272 & 7 \\
181 & 128 & 206 & 25 & 231 & 3 & 276 & 4 \\
182 & 207 & 207 & 13 & 232 & 21 & 280 & 4 \\
183 & 109 & 208 & 59 & 234 & 10 & 284 & 3 \\
184 & 155 & 209 & 4 & 235 & 1 & 288 & 5 \\
185 & 75 & 210 & 51 & 236 & 26 & 294 & 1 \\
186 & 149 & 211 & 2 & 238 & 5 & 300 & 1 \\
187 & 57 & 212 & 46 & 239 & 1 & 303 & 1 \\
188 & 159 & 213 & 10 & 240 & 26 & 308 & 1 \\
189 & 45 & 214 & 14 & 242 & 1 & 312 & 3 \\
190 & 91 & 215 & 2 & 243 & 1 & 320 & 2 \\
191 & 44 & 216 & 38 & 244 & 7 & 336 & 2 \\
192 & 123 & 217 & 3 & 245 & 1 & 384 & 1 \\
193 & 36 & 218 & 15 & 248 & 11 & & \\
\hline & & & & & &
\end{tabular}


Table 10: Number of prisms

\begin{tabular}{rrrrrrrr}
\hline Prism & $\#$ & Prism & $\#$ & Prism & \# & Prism & $\#$ \\
\hline 171 & 1 & 250 & 75976 & 287 & 42388161 & 324 & 198341505 \\
189 & 1 & 251 & 98127 & 288 & 46639711 & 325 & 196983412 \\
200 & 1 & 252 & 125286 & 289 & 51169522 & 326 & 195225803 \\
207 & 1 & 253 & 158108 & 290 & 55931715 & 327 & 193085136 \\
211 & 1 & 254 & 200729 & 291 & 60918787 & 328 & 190605951 \\
216 & 2 & 255 & 253967 & 292 & 66151873 & 329 & 187795686 \\
217 & 1 & 256 & 318185 & 293 & 71586084 & 330 & 184649280 \\
219 & 6 & 257 & 397908 & 294 & 77237835 & 331 & 181212592 \\
221 & 1 & 258 & 492617 & 295 & 83032700 & 332 & 177549753 \\
222 & 6 & 259 & 610716 & 296 & 88988957 & 333 & 173586201 \\
223 & 17 & 260 & 753345 & 297 & 95089060 & 334 & 169440136 \\
224 & 22 & 261 & 921675 & 298 & 101293200 & 335 & 165109202 \\
225 & 27 & 262 & 1126793 & 299 & 107579627 & 336 & 160640418 \\
226 & 25 & 263 & 1368838 & 300 & 113892453 & 337 & 155982892 \\
227 & 41 & 264 & 1655279 & 301 & 120225453 & 338 & 151293063 \\
228 & 73 & 265 & 1993377 & 302 & 126496164 & 339 & 146440917 \\
229 & 130 & 266 & 2390574 & 303 & 132753692 & 340 & 141569668 \\
230 & 166 & 267 & 2851791 & 304 & 138902842 & 341 & 136664720 \\
231 & 245 & 268 & 3389099 & 305 & 144926038 & 342 & 131727398 \\
232 & 321 & 269 & 4010807 & 306 & 150790370 & 343 & 126770273 \\
233 & 448 & 270 & 4727106 & 307 & 156429753 & 344 & 121858346 \\
234 & 667 & 271 & 5547565 & 308 & 161884623 & 345 & 116981409 \\
235 & 932 & 272 & 6485240 & 309 & 167038214 & 346 & 112190976 \\
236 & 1291 & 273 & 7552715 & 310 & 171888128 & 347 & 107410238 \\
237 & 1750 & 274 & 8757871 & 311 & 176448741 & 348 & 102737476 \\
238 & 2462 & 275 & 10118769 & 312 & 180620616 & 349 & 98136704 \\
239 & 3344 & 276 & 11640128 & 313 & 184476735 & 350 & 93657722 \\
240 & 4558 & 277 & 13335175 & 314 & 187911346 & 351 & 89292744 \\
241 & 6221 & 278 & 15233835 & 315 & 190927860 & 352 & 85046857 \\
242 & 8341 & 279 & 17317913 & 316 & 193530670 & 353 & 80920249 \\
243 & 11120 & 280 & 19617190 & 317 & 195702979 & 354 & 76911822 \\
244 & 14888 & 281 & 22137761 & 318 & 197395867 & 355 & 73054525 \\
245 & 20119 & 282 & 24884491 & 319 & 198675356 & 356 & 69332115 \\
246 & 26400 & 283 & 27887561 & 320 & 199497261 & 357 & 65735409 \\
247 & 34577 & 284 & 31140015 & 321 & 199874535 & 358 & 62291346 \\
248 & 44753 & 285 & 34623522 & 322 & 199760946 & 359 & 58986226 \\
249 & 58845 & 286 & 38376738 & 323 & 199286571 & 360 & 55805608 \\
\hline & & & & & & & \\
& & & & &
\end{tabular}


Table 10: Number of prisms (cont.)

\begin{tabular}{rrrrrrrr}
\hline Prism & $\#$ & Prism & $\#$ & Prism & $\#$ & Prism & $\#$ \\
\hline 361 & 52776788 & 398 & 5210998 & 435 & 424445 & 472 & 95566 \\
362 & 49877144 & 399 & 4870806 & 436 & 400992 & 473 & 92467 \\
363 & 47109094 & 400 & 4555184 & 437 & 375930 & 474 & 89604 \\
364 & 44477939 & 401 & 4255687 & 438 & 356584 & 475 & 86116 \\
365 & 41956665 & 402 & 3975185 & 439 & 335932 & 476 & 83388 \\
366 & 39596950 & 403 & 3710635 & 440 & 318533 & 477 & 80516 \\
367 & 37316718 & 404 & 3468155 & 441 & 300617 & 478 & 78206 \\
368 & 35158337 & 405 & 3235022 & 442 & 286646 & 479 & 74644 \\
369 & 33131446 & 406 & 3021856 & 443 & 271545 & 480 & 72289 \\
370 & 31199621 & 407 & 2817205 & 444 & 258555 & 481 & 68924 \\
371 & 29360909 & 408 & 2632611 & 445 & 245429 & 482 & 67293 \\
372 & 27626089 & 409 & 2454635 & 446 & 235409 & 483 & 63891 \\
373 & 25997783 & 410 & 2292545 & 447 & 224067 & 484 & 62065 \\
374 & 24455068 & 411 & 2137919 & 448 & 214575 & 485 & 58964 \\
375 & 22993528 & 412 & 1995564 & 449 & 205399 & 486 & 56790 \\
376 & 21604049 & 413 & 1861521 & 450 & 197610 & 487 & 54505 \\
377 & 20310057 & 414 & 1737449 & 451 & 188729 & 488 & 52492 \\
378 & 19075074 & 415 & 1616932 & 452 & 182542 & 489 & 49354 \\
379 & 17916453 & 416 & 1509591 & 453 & 176060 & 490 & 47536 \\
380 & 16819109 & 417 & 1404929 & 454 & 168815 & 491 & 45253 \\
381 & 15795662 & 418 & 1314772 & 455 & 162976 & 492 & 43832 \\
382 & 14826839 & 419 & 1225935 & 456 & 158019 & 493 & 40816 \\
383 & 13907432 & 420 & 1144721 & 457 & 152147 & 494 & 39536 \\
384 & 13050725 & 421 & 1067065 & 458 & 148600 & 495 & 37181 \\
385 & 12241906 & 422 & 995655 & 459 & 142312 & 496 & 35949 \\
386 & 11482906 & 423 & 927859 & 460 & 138498 & 497 & 33708 \\
387 & 10762834 & 424 & 868000 & 461 & 134174 & 498 & 32268 \\
388 & 10084561 & 425 & 811642 & 462 & 130272 & 499 & 30063 \\
389 & 9453238 & 426 & 758276 & 463 & 125969 & 500 & 28901 \\
390 & 8853538 & 427 & 709328 & 464 & 122632 & 501 & 27030 \\
391 & 8294860 & 428 & 663317 & 465 & 117860 & 502 & 25906 \\
392 & 7771024 & 429 & 619097 & 466 & 115901 & 503 & 24000 \\
393 & 7269785 & 430 & 582159 & 467 & 111021 & 504 & 23162 \\
394 & 6806485 & 431 & 544981 & 468 & 108594 & 505 & 21754 \\
395 & 6363581 & 432 & 513193 & 469 & 104985 & 506 & 20937 \\
396 & 5960984 & 433 & 479631 & 470 & 101572 & 507 & 19322 \\
397 & 5569324 & 434 & 452765 & 471 & 98344 & 508 & 18497 \\
\hline & & & & & & &
\end{tabular}


Table 10: Number of prisms (cont.)

\begin{tabular}{rrrrrrrr}
\hline Prism & $\#$ & Prism & $\#$ & Prism & $\#$ & Prism & $\#$ \\
\hline 509 & 17095 & 546 & 1731 & 583 & 334 & 620 & 2116 \\
510 & 16519 & 547 & 1499 & 584 & 421 & 621 & 2254 \\
511 & 15154 & 548 & 1394 & 585 & 413 & 622 & 2301 \\
512 & 14143 & 549 & 1222 & 586 & 434 & 623 & 2357 \\
513 & 13411 & 550 & 1291 & 587 & 392 & 624 & 2510 \\
514 & 12808 & 551 & 1103 & 588 & 480 & 625 & 2523 \\
515 & 11849 & 552 & 1094 & 589 & 420 & 626 & 2527 \\
516 & 11530 & 553 & 926 & 590 & 465 & 627 & 2581 \\
517 & 10468 & 554 & 1000 & 591 & 474 & 628 & 2719 \\
518 & 10064 & 555 & 826 & 592 & 572 & 629 & 2826 \\
519 & 9280 & 556 & 885 & 593 & 521 & 630 & 2966 \\
520 & 8869 & 557 & 719 & 594 & 593 & 631 & 3099 \\
521 & 8064 & 558 & 757 & 595 & 599 & 632 & 3144 \\
522 & 7774 & 559 & 648 & 596 & 662 & 633 & 3059 \\
523 & 7153 & 560 & 728 & 597 & 647 & 634 & 3157 \\
524 & 6714 & 561 & 532 & 598 & 710 & 635 & 3236 \\
525 & 6300 & 562 & 629 & 599 & 729 & 636 & 3362 \\
526 & 6014 & 563 & 517 & 600 & 830 & 637 & 3384 \\
527 & 5362 & 564 & 511 & 601 & 872 & 638 & 3465 \\
528 & 5209 & 565 & 436 & 602 & 972 & 639 & 3487 \\
529 & 4847 & 566 & 505 & 603 & 959 & 640 & 3393 \\
530 & 4551 & 567 & 416 & 604 & 1011 & 641 & 3423 \\
531 & 4184 & 568 & 497 & 605 & 1050 & 642 & 3599 \\
532 & 4108 & 569 & 374 & 606 & 1149 & 643 & 3580 \\
533 & 3736 & 570 & 452 & 607 & 1188 & 644 & 3753 \\
534 & 3743 & 571 & 358 & 608 & 1375 & 645 & 3622 \\
535 & 3116 & 572 & 387 & 609 & 1308 & 646 & 3827 \\
536 & 3141 & 573 & 349 & 610 & 1358 & 647 & 3643 \\
537 & 2792 & 574 & 345 & 611 & 1471 & 648 & 3812 \\
538 & 2744 & 575 & 330 & 612 & 1495 & 649 & 3744 \\
539 & 2548 & 576 & 381 & 613 & 1553 & 650 & 3902 \\
540 & 2452 & 577 & 336 & 614 & 1701 & 651 & 3579 \\
541 & 2100 & 578 & 351 & 615 & 1703 & 652 & 3790 \\
542 & 2155 & 579 & 326 & 616 & 1875 & 653 & 3752 \\
543 & 1864 & 580 & 382 & 617 & 1868 & 654 & 3713 \\
544 & 1844 & 581 & 315 & 618 & 1980 & 655 & 3683 \\
545 & 1613 & 582 & 399 & 619 & 2027 & 656 & 3662 \\
\hline & & & & & & &
\end{tabular}


Table 10: Number of prisms (cont.)

\begin{tabular}{rrrrrrrr}
\hline Prism & $\#$ & Prism & $\#$ & Prism & $\#$ & Prism & $\#$ \\
\hline 657 & 3649 & 694 & 1495 & 731 & 194 & 768 & 27 \\
658 & 3597 & 695 & 1380 & 732 & 211 & 769 & 22 \\
659 & 3637 & 696 & 1400 & 733 & 175 & 770 & 21 \\
660 & 3667 & 697 & 1250 & 734 & 177 & 771 & 14 \\
661 & 3567 & 698 & 1324 & 735 & 164 & 772 & 21 \\
662 & 3416 & 699 & 1141 & 736 & 152 & 773 & 12 \\
663 & 3464 & 700 & 1136 & 737 & 154 & 774 & 24 \\
664 & 3326 & 701 & 1010 & 738 & 147 & 775 & 10 \\
665 & 3370 & 702 & 1024 & 739 & 116 & 776 & 16 \\
666 & 3370 & 703 & 931 & 740 & 116 & 777 & 5 \\
667 & 3294 & 704 & 935 & 741 & 88 & 778 & 13 \\
668 & 3155 & 705 & 833 & 742 & 123 & 779 & 3 \\
669 & 3170 & 706 & 844 & 743 & 89 & 780 & 5 \\
670 & 3123 & 707 & 729 & 744 & 97 & 781 & 8 \\
671 & 3023 & 708 & 759 & 745 & 75 & 782 & 10 \\
672 & 3036 & 709 & 669 & 746 & 103 & 783 & 6 \\
673 & 2903 & 710 & 666 & 747 & 68 & 784 & 9 \\
674 & 2895 & 711 & 636 & 748 & 90 & 785 & 5 \\
675 & 2735 & 712 & 624 & 749 & 79 & 786 & 10 \\
676 & 2797 & 713 & 597 & 750 & 91 & 787 & 5 \\
677 & 2606 & 714 & 564 & 751 & 56 & 788 & 9 \\
678 & 2600 & 715 & 511 & 752 & 60 & 789 & 2 \\
679 & 2416 & 716 & 531 & 753 & 44 & 790 & 8 \\
680 & 2493 & 717 & 433 & 754 & 65 & 791 & 8 \\
681 & 2302 & 718 & 455 & 755 & 43 & 792 & 12 \\
682 & 2238 & 719 & 439 & 756 & 45 & 793 & 1 \\
683 & 2215 & 720 & 394 & 757 & 46 & 795 & 2 \\
684 & 2072 & 721 & 359 & 758 & 39 & 796 & 2 \\
685 & 2115 & 722 & 366 & 759 & 42 & 797 & 2 \\
686 & 2023 & 723 & 334 & 760 & 35 & 798 & 3 \\
687 & 1880 & 724 & 326 & 761 & 28 & 799 & 1 \\
688 & 1868 & 725 & 262 & 762 & 30 & 800 & 2 \\
689 & 1724 & 726 & 306 & 763 & 23 & 801 & 1 \\
690 & 1645 & 727 & 229 & 764 & 40 & 805 & 1 \\
691 & 1620 & 728 & 253 & 765 & 15 & 806 & 5 \\
692 & 1595 & 729 & 253 & 766 & 16 & 807 & 1 \\
693 & 1497 & 730 & 218 & 767 & 19 & 808 & 4 \\
\hline & & & & & & &
\end{tabular}


Table 10: Number of prisms (cont.)

\begin{tabular}{rrrrrrrr}
\hline Prism & \# & Prism & $\#$ & Prism & \# & Prism & \# \\
\hline 809 & 1 & 838 & 1 & 856 & 1 & 912 & 2 \\
814 & 1 & 840 & 2 & 864 & 2 & 918 & 6 \\
816 & 3 & 844 & 1 & 868 & 1 & 1152 & 3 \\
818 & 1 & 846 & 2 & 870 & 4 & & \\
822 & 14 & 850 & 1 & 878 & 1 & & \\
832 & 1 & 852 & 1 & 888 & 2 & & \\
\hline
\end{tabular}

Table 11: Number of mitres

\begin{tabular}{rrrrrrrr}
\hline Mitre & $\#$ & Mitre & $\#$ & Mitre & $\#$ & Mitre & $\#$ \\
\hline 0 & 4 & 29 & 666856068 & 56 & 699975 & 83 & 39 \\
3 & 11 & 30 & 726726670 & 57 & 427224 & 84 & 83 \\
4 & 27 & 31 & 765630873 & 58 & 261965 & 85 & 16 \\
5 & 94 & 32 & 780912655 & 59 & 162576 & 86 & 47 \\
6 & 463 & 33 & 771673239 & 60 & 105125 & 87 & 20 \\
7 & 1587 & 34 & 739625001 & 61 & 68560 & 88 & 34 \\
8 & 5196 & 35 & 688305207 & 62 & 47177 & 89 & 7 \\
9 & 16130 & 36 & 622481814 & 63 & 32413 & 90 & 54 \\
10 & 45051 & 37 & 547576707 & 64 & 23643 & 91 & 1 \\
11 & 119156 & 38 & 468917351 & 65 & 16778 & 92 & 19 \\
12 & 292925 & 39 & 391303591 & 66 & 12393 & 93 & 9 \\
13 & 685985 & 40 & 318424938 & 67 & 8661 & 94 & 7 \\
14 & 1502196 & 41 & 252876637 & 68 & 6489 & 96 & 27 \\
15 & 3122990 & 42 & 196124480 & 69 & 4295 & 98 & 2 \\
16 & 6160011 & 43 & 148685094 & 70 & 3264 & 99 & 2 \\
17 & 11527121 & 44 & 110224646 & 71 & 2181 & 100 & 6 \\
18 & 20542885 & 45 & 79959174 & 72 & 1700 & 102 & 7 \\
19 & 34903297 & 46 & 56803086 & 73 & 990 & 104 & 2 \\
20 & 56577514 & 47 & 39545210 & 74 & 909 & 105 & 2 \\
21 & 87700390 & 48 & 26981662 & 75 & 469 & 108 & 5 \\
22 & 130128895 & 49 & 18067853 & 76 & 465 & 112 & 3 \\
23 & 185013010 & 50 & 11873632 & 77 & 270 & 114 & 1 \\
24 & 252364501 & 51 & 7665089 & 78 & 263 & 116 & 2 \\
25 & 330721805 & 52 & 4870654 & 79 & 122 & 120 & 2 \\
26 & 416700734 & 53 & 3046823 & 80 & 191 & 144 & 3 \\
27 & 505540524 & 54 & 1883004 & 81 & 72 & & \\
28 & 591121831 & 55 & 1150672 & 82 & 96 & & \\
\hline & & & & & & &
\end{tabular}


Table 12: Number of hexagons

\begin{tabular}{rrrrrrrr}
\hline Hexa & $\#$ & Hexa & $\#$ & Hexa & $\#$ & Hexa & $\#$ \\
\hline 0 & 1 & 34 & 724247745 & 66 & 436234 & 98 & 110 \\
2 & 1 & 35 & 714131642 & 67 & 326333 & 99 & 62 \\
4 & 8 & 36 & 685867252 & 68 & 239208 & 100 & 77 \\
5 & 2 & 37 & 642422184 & 69 & 179527 & 101 & 33 \\
6 & 18 & 38 & 587540455 & 70 & 134495 & 102 & 74 \\
7 & 42 & 39 & 525307321 & 71 & 100405 & 103 & 17 \\
8 & 275 & 40 & 459726499 & 72 & 75980 & 104 & 35 \\
9 & 1060 & 41 & 394271746 & 73 & 57056 & 105 & 28 \\
10 & 3888 & 42 & 331862444 & 74 & 43803 & 106 & 28 \\
11 & 13543 & 43 & 274475233 & 75 & 31922 & 107 & 10 \\
12 & 42046 & 44 & 223366811 & 76 & 26629 & 108 & 136 \\
13 & 119420 & 45 & 179088397 & 77 & 17366 & 109 & 10 \\
14 & 315586 & 46 & 141683536 & 78 & 13996 & 110 & 17 \\
15 & 769997 & 47 & 110703052 & 79 & 9867 & 111 & 10 \\
16 & 1750488 & 48 & 85587484 & 80 & 8815 & 112 & 14 \\
17 & 3711050 & 49 & 65546910 & 81 & 5888 & 113 & 1 \\
18 & 7390282 & 50 & 49813749 & 82 & 5139 & 114 & 17 \\
19 & 13851974 & 51 & 37586617 & 83 & 3120 & 115 & 1 \\
20 & 24536316 & 52 & 28199864 & 84 & 2880 & 116 & 18 \\
21 & 41147211 & 53 & 21046347 & 85 & 1883 & 117 & 4 \\
22 & 65593940 & 54 & 15677184 & 86 & 2264 & 118 & 1 \\
23 & 99604643 & 55 & 11622883 & 87 & 1127 & 120 & 10 \\
24 & 144448598 & 56 & 8623668 & 88 & 1016 & 121 & 1 \\
25 & 200532422 & 57 & 6370044 & 89 & 615 & 122 & 4 \\
26 & 266967992 & 58 & 4713086 & 90 & 1645 & 124 & 8 \\
27 & 341559277 & 59 & 3483045 & 91 & 436 & 126 & 16 \\
28 & 420712045 & 60 & 2580662 & 92 & 408 & 128 & 1 \\
29 & 499765074 & 61 & 1909874 & 93 & 249 & 132 & 3 \\
30 & 573401076 & 62 & 1419396 & 94 & 234 & 144 & 12 \\
31 & 636579383 & 63 & 1050752 & 95 & 150 & 171 & 1 \\
32 & 684620989 & 64 & 786486 & 96 & 248 & & \\
33 & 714416762 & 65 & 577280 & 97 & 75 & & \\
\hline & & & & & & &
\end{tabular}


Table 13: Number of crowns

\begin{tabular}{rrrrrrrr}
\hline Crown & \# & Crown & $\#$ & Crown & $\#$ & Crown & $\#$ \\
\hline 0 & 4 & 75 & 84 & 112 & 39276 & 149 & 11026967 \\
24 & 8 & 76 & 225 & 113 & 46162 & 150 & 12418598 \\
28 & 1 & 77 & 98 & 114 & 55376 & 151 & 13951975 \\
32 & 7 & 78 & 230 & 115 & 65172 & 152 & 15649589 \\
34 & 1 & 79 & 146 & 116 & 78169 & 153 & 17504989 \\
36 & 17 & 80 & 303 & 117 & 92109 & 154 & 19550198 \\
40 & 4 & 81 & 184 & 118 & 110533 & 155 & 21784052 \\
42 & 2 & 82 & 352 & 119 & 130711 & 156 & 24217202 \\
44 & 3 & 83 & 271 & 120 & 155188 & 157 & 26857968 \\
45 & 1 & 84 & 507 & 121 & 183383 & 158 & 29735229 \\
46 & 3 & 85 & 409 & 122 & 218318 & 159 & 32838784 \\
48 & 17 & 86 & 625 & 123 & 256913 & 160 & 36187030 \\
49 & 2 & 87 & 538 & 124 & 304546 & 161 & 39768756 \\
50 & 4 & 88 & 788 & 125 & 357058 & 162 & 43644429 \\
51 & 3 & 89 & 745 & 126 & 420855 & 163 & 47762633 \\
52 & 19 & 90 & 1103 & 127 & 493066 & 164 & 52146277 \\
54 & 23 & 91 & 997 & 128 & 580012 & 165 & 56809902 \\
55 & 5 & 92 & 1448 & 129 & 678149 & 166 & 61761576 \\
56 & 37 & 93 & 1460 & 130 & 794787 & 167 & 66960296 \\
57 & 9 & 94 & 1941 & 131 & 925609 & 168 & 72455628 \\
58 & 16 & 95 & 1999 & 132 & 1080365 & 169 & 78205211 \\
59 & 6 & 96 & 2809 & 133 & 1256516 & 170 & 84194952 \\
60 & 72 & 97 & 2861 & 134 & 1462493 & 171 & 90422800 \\
61 & 5 & 98 & 3569 & 135 & 1691178 & 172 & 96907778 \\
62 & 22 & 99 & 3832 & 136 & 1960531 & 173 & 103579676 \\
63 & 16 & 100 & 5157 & 137 & 2262445 & 174 & 110428354 \\
64 & 65 & 101 & 5644 & 138 & 2612802 & 175 & 117487564 \\
65 & 16 & 102 & 7012 & 139 & 3008486 & 176 & 124638538 \\
66 & 77 & 103 & 7868 & 140 & 3455009 & 177 & 131927624 \\
67 & 19 & 104 & 9735 & 141 & 3958995 & 178 & 139275613 \\
68 & 71 & 105 & 11111 & 142 & 4536189 & 179 & 146638317 \\
69 & 32 & 106 & 13806 & 143 & 5178047 & 180 & 154028623 \\
70 & 81 & 107 & 15906 & 144 & 5903381 & 181 & 161359146 \\
71 & 55 & 108 & 19655 & 145 & 6715687 & 182 & 168619294 \\
72 & 173 & 109 & 22619 & 146 & 7629172 & 183 & 175716385 \\
73 & 65 & 110 & 27800 & 147 & 8645817 & 184 & 182665320 \\
74 & 144 & 111 & 32269 & 148 & 9772477 & 185 & 189374242 \\
\hline
\end{tabular}


Table 13: Number of crowns (cont.)

\begin{tabular}{rrrrrrrr}
\hline Crown & \# & Crown & \# & Crown & \# & Crown & $\#$ \\
\hline 186 & 195806871 & 217 & 134563689 & 248 & 4411819 & 279 & 3866 \\
187 & 201907700 & 218 & 126661383 & 249 & 3734688 & 280 & 3026 \\
188 & 207659159 & 219 & 118861873 & 250 & 3149311 & 281 & 2220 \\
189 & 212988838 & 220 & 111206681 & 251 & 2648386 & 282 & 1621 \\
190 & 217852023 & 221 & 103671809 & 252 & 2219528 & 283 & 1190 \\
191 & 222201411 & 222 & 96346526 & 253 & 1850527 & 284 & 880 \\
192 & 226058774 & 223 & 89252032 & 254 & 1538216 & 285 & 617 \\
193 & 229322865 & 224 & 82406974 & 255 & 1272656 & 286 & 458 \\
194 & 231961935 & 225 & 75860206 & 256 & 1051337 & 287 & 337 \\
195 & 233966564 & 226 & 69578295 & 257 & 862379 & 288 & 237 \\
196 & 235362932 & 227 & 63614491 & 258 & 707331 & 289 & 186 \\
197 & 236055372 & 228 & 57977229 & 259 & 576064 & 290 & 135 \\
198 & 236115675 & 229 & 52664490 & 260 & 468744 & 291 & 88 \\
199 & 235469719 & 230 & 47671940 & 261 & 378298 & 292 & 63 \\
200 & 234145518 & 231 & 43011588 & 262 & 304621 & 293 & 35 \\
201 & 232142509 & 232 & 38676935 & 263 & 244241 & 294 & 36 \\
202 & 229517435 & 233 & 34668107 & 264 & 194690 & 295 & 12 \\
203 & 226209636 & 234 & 30961644 & 265 & 155113 & 296 & 19 \\
204 & 222338699 & 235 & 27551781 & 266 & 123781 & 297 & 14 \\
205 & 217827123 & 236 & 24435171 & 267 & 96942 & 298 & 5 \\
206 & 212820389 & 237 & 21602222 & 268 & 76095 & 299 & 7 \\
207 & 207301265 & 238 & 19035194 & 269 & 59785 & 300 & 4 \\
208 & 201303814 & 239 & 16706493 & 270 & 46762 & 301 & 1 \\
209 & 194883375 & 240 & 14612461 & 271 & 36086 & 302 & 1 \\
210 & 188122519 & 241 & 12739285 & 272 & 27879 & 303 & 4 \\
211 & 180992703 & 242 & 11064520 & 273 & 21426 & 306 & 1 \\
212 & 173617922 & 243 & 9577688 & 274 & 16461 & 309 & 2 \\
213 & 166018485 & 244 & 8260815 & 275 & 12570 & 314 & 1 \\
214 & 158267357 & 245 & 7095407 & 276 & 9619 & & \\
215 & 150399412 & 246 & 6078552 & 277 & 7117 & & \\
216 & 142486139 & 247 & 5188692 & 278 & 5272 & & \\
\hline & & & & & & &
\end{tabular}

